\title{
Superior Vena Cava Thrombosis Due to a Temporary Hemodialysis Catheter Presenting After 5 Years With Hematemesis, Hemoptysis and Pleural Effusion
}

\author{
Nidal Arnous ${ }^{\mathrm{a}, \mathrm{c}}$, Munaza Akunjee ${ }^{\mathrm{b}}$
}

\begin{abstract}
Superior vena cava (SVC) thrombosis is increasingly seen now due to the liberal use of intravascular catheters. Most of these cases present with symptoms acutely, like any case of deep vein thrombosis, usually with ipsilateral arm and face swelling. Our patient presented after 5 years with unusual symptoms that are unrelated to the thrombosis itself, but due to the collateral circulation. He was a 38 -year-old male patient with end-stage renal disease on hemodialysis (HD) for 9 years, initially via a left arm arteriovenous (AV) fistula, then via a temporary right subclavian dialysis catheter for a period of 5 months before being maintained on his current AV fistula in the right arm for the last 5 years. He presented with intermittent episodes of hematemesis and hemoptysis, and was also found to have pleural effusion. He was found to have chronic thrombosis in the SVC. Chronic SVC obstruction causes formation and increase in the flow in the collateral circulation in the esophageal plexus causing varices, in the intercostals veins causing pleural fluid formation, and in the bronchial veins leading to hemoptysis. The AV fistula aggravated these findings by further increasing the flow and the pressure in the collateral circulation. In conclusion, chronic SVC thrombosis can occur as a consequence of HD catheters, and may present with symptoms related to the collateral circulation.
\end{abstract}

Keywords: Superior vena cava; ESRD; Thrombosis; Catheter

\section{Introduction}

Dialysis catheters can cause many complications. Superior vena cava (SVC) thrombosis is a rare complication, but in-

Manuscript accepted for publication August 18, 2014

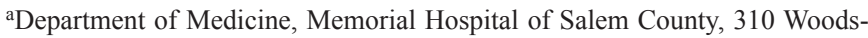
town Rd, Salem, NJ 08079, USA

bDepartment of Medicine, Providence Hospital, 1150 Varnum St, Washington, DC 20017, USA

${ }^{\mathrm{c} C o r r e s p o n d i n g ~ A u t h o r: ~ N i d a l ~ A r n o u s, ~ D e p a r t m e n t ~ o f ~ M e d i c i n e, ~ M e m o-~}$ rial Hospital of Salem County, 310 Woodstown Rd, Salem, NJ 08079, USA. Email:nidal.arnous@hotmail.com

doi: http://dx.doi.org/10.14740/jmc1925w creasingly seen due to the liberal use of dialysis catheters [1]. We describe a case of chronic thrombosis in the SVC as a complication of a right subclavian hemodialysis (HD) catheter, which presented 5 years after its temporary placement without the typical symptoms of acute SVC obstruction.

\section{Case Report}

A 38-year-old male with hypertension and end-stage renal disease (ESRD) on HD presented with intermittent episodes of hemoptysis and hematemesis for a period of 1 month. His dialysis was started 9 years ago, initially via a left arm arteriovenous (AV) fistula, and then temporarily via a right subclavian catheter for a period of 5 months when the fistula was not functioning, then via a right arm AV fistula for the last 5 years. He did not have any vascular intervention over the last 5 years. He is a smoker (15 pack-year), with no significant alcohol use. He was hemodynamically stable with no orthostatic changes. He was afebrile, and oxygen saturation of $98 \%$ on room air. His examination was significant for multiple dilated, tortuous veins over the chest and abdominal walls and on the upper back (Fig. 1), which as per the patient, they have been developing gradually over the last few years. Chest examination showed decreased breath sounds at the bases of both lungs.

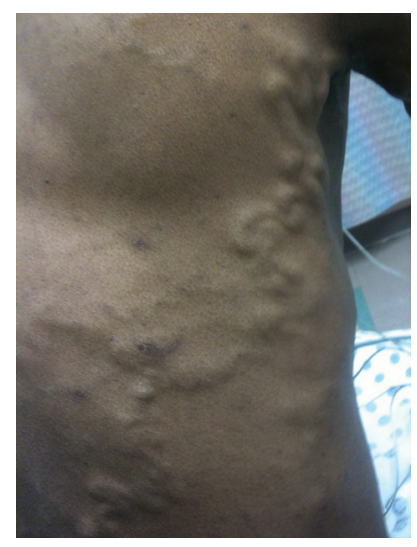

Figure 1. Picture of the patient's lateral chest wall showing dilated, tortuous veins. 


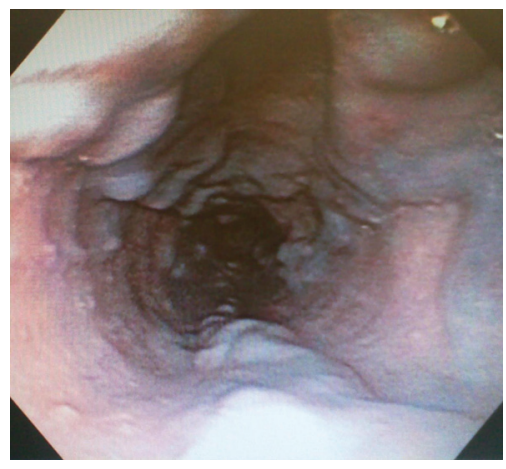

Figure 2. Upper endoscopy (EGD) showing grade 4 varices in the middle and lower portions of the esophagus.

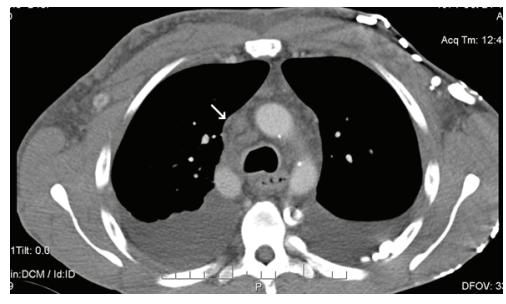

Figure 3. Computerized tomography of the chest showing a filling defect in the superior vena cava (arrow).

\section{Investigations}

CBC showed only mild anemia (hemoglobin $11.9 \mathrm{~g} / \mathrm{dL}$, which was stable during hospitalization), and the platelet count was normal. His coagulation profile was normal. Esophagogastroduodenoscopy (EGD) (Fig. 2) showed grade 4 esophageal varices in the middle and lower thirds of the esophagus, with no active bleeding. Workup for any underlying liver disease was negative, with normal liver function tests, hepatitis screening, and ultrasound and CT scanning of the liver. Chest X-ray showed obliteration of both costophrenic angles. CT scan of chest (Fig. 3) revealed bilateral pleural effusion. A filling defect was also seen in the SVC, in addition to thoracic varices which were also involving the azygos vein. Straw-colored transudative fluid $950 \mathrm{~mL}$ was drained by thoracentesis from the right pleural effusion. LDH was $102 \mathrm{IU} / \mathrm{L}$ (serum 232 $\mathrm{IU} / \mathrm{L}$, ratio is 0.44 ), glucose was $96 \mathrm{mg} / \mathrm{dL}$, and total protein was $2.8 \mathrm{~g} / \mathrm{dL}$ (serum $8.6 \mathrm{~g} / \mathrm{dL}$ ). Cytology, AFB staining and cultures were all negative. Purified protein derivative skin testing was negative. Bronchoscopy was normal. Echocardiogram was normal. Venogram (Fig. 4) for the proximal right upper extremity and neck showed extensive thrombosis involving the proximal right subclavian vein with complete occlusion, extending into the SVC. It also showed extensive collaterals between right axillary and distal subclavian veins and the right cervical veins including jugular veins. The clinical and radiographic findings were consistent with chronic SVC thrombosis completely obstructing its lumen, and extensive to the point of obstructing azygos flow into the SVC. Thrombophilia workup was done subsequently and was negative (including protein C,

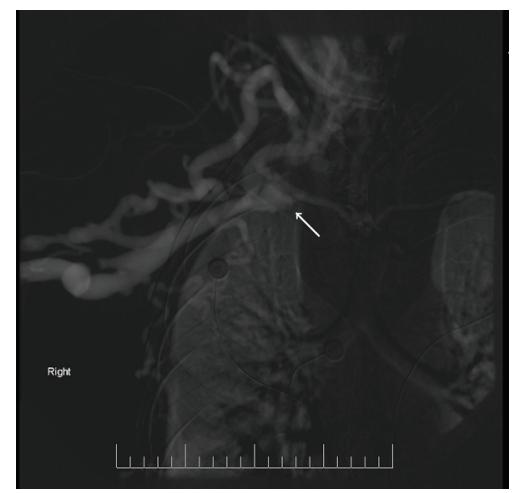

Figure 4. Venogram for the proximal right upper extremity and the neck, showing complete occlusion of the proximal right subclavian artery (arrow) with extensive collaterals.

protein S, antithrombin III, factor V Leiden, prothrombin gene mutation, antiphospholipid antibodies, factor VIII excess, and hyperhomocysteinemia).

\section{Course}

As the patient remained stable with no further episodes of bleeding, he declined a bypass procedure for SVC. We discussed with him the option of closing the right upper arm AV fistula as a temporizing measure and creating an AV fistula in the femoral area, in an effort to try to decrease the pressure inside the collateral circulation. He agreed for that and had an AV fistula created in his left femoral area, and was discharged from the hospital after the procedure to follow up in a few weeks for closure of his right arm fistula. Unfortunately the patient presented 3 weeks later with a sudden episode of massive hemoptysis. He had cardiac arrest and could not be resuscitated.

\section{Discussion}

Catheter-induced trauma to the venous endothelium at the time of placement may be a triggering factor for thrombosis [1]. Subclavian vein thrombosis, when symptomatic, commonly presents with florid symptoms and signs such as ipsilateral arm edema and pain around the shoulder area. Thrombosis that involves or extends into the SVC is rare, and usually manifests with face edema as well. Most of these cases present in the presence of the catheter or within a period of weeks after removal [2-4]. The patient in our case presented after 5 years and did not have these symptoms because of the slowly progressing thrombosis with gradual obstruction to the lumen and simultaneous development of collaterals.

Pleural fluid formation and resorption occur at the surface of the parietal pleura. Venous drainage from the parietal pleura is via intercostal veins. Most posterior intercostal veins drain into the azygos system (Fig. 5). SVC thrombosis, in association with the high flow from the AV fistula, increases the pressure in the intercostal veins, which causes excess pleural fluid 


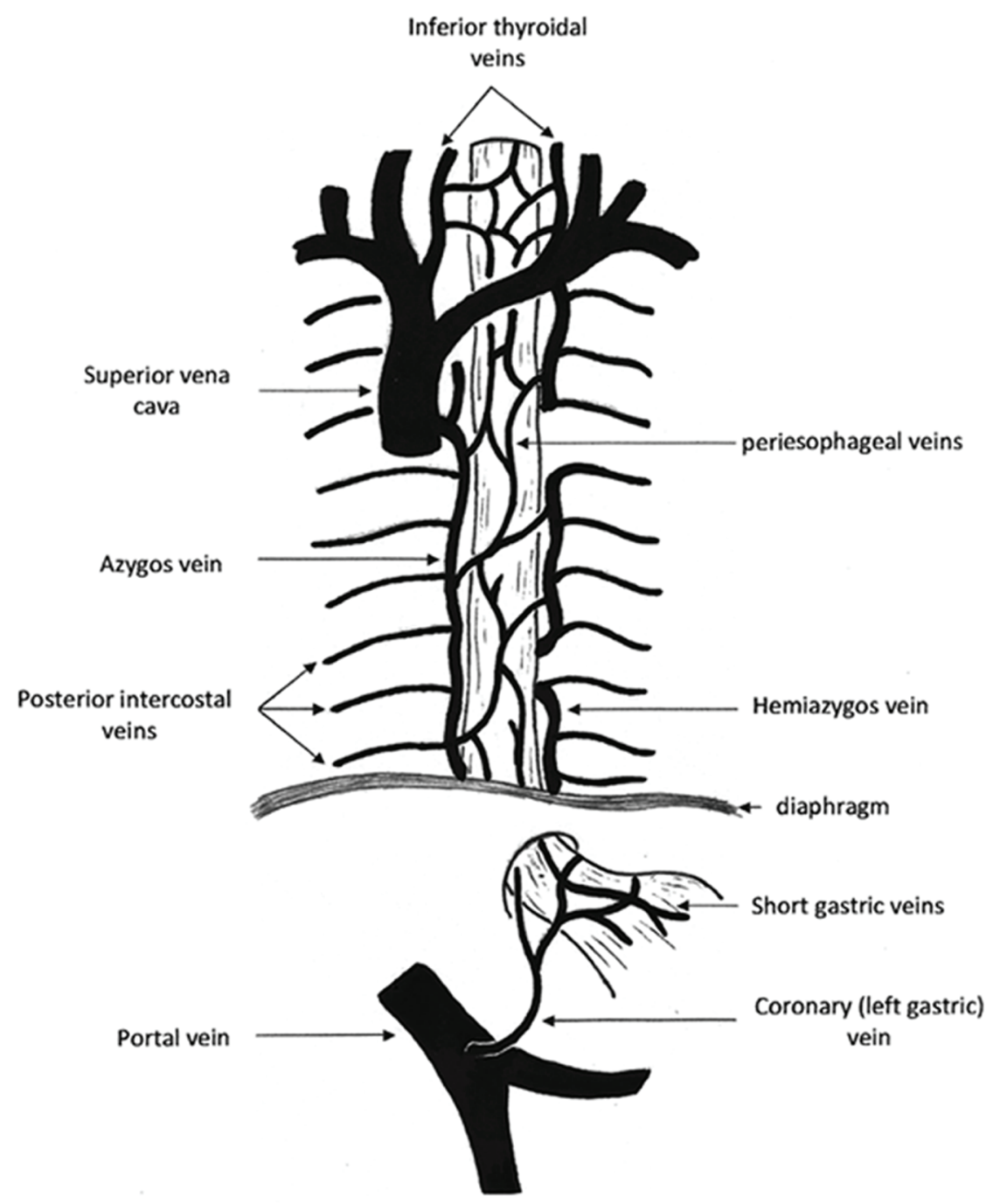

Figure 5. Veins draining into the azygos system and then into the superior vena cava.

formation, and also impedes pleural fluid resorption [5].

Esophageal varices can also occur in patients with SVC obstruction. The majority of blood from the esophagus is drained via the esophageal veins to the azygos (Fig. 5). Obstruction of this venous flow to the SVC will increase the pressure in the esophageal veins and can result in the development of "downhill" esophageal varices [6-9]. Hemoptysis is also due to increased pressures in the bronchial veins which normally drain into azygos system [10].

Management should be individualized. Anticoagulation and thrombolysis may have a role. Our case was chronic and delayed; therefore there is no role for anticoagulation or thrombolysis. Interventional procedures include balloon angioplasty and stent placement, with variable long-term patency due to restenosis [11]. This was not an option in our case due to complete obstruction of the SVC lumen. SVC bypass surgery has also been used successfully; however, our patient declined this intervention [12]. In patients with ESRD on HD, closure of the $\mathrm{AV}$ fistula is a logical intervention if the patient is not suitable for any of the above treatment options.

\section{Conclusion}

Chronic thrombosis of the SVC can occur as a consequence of HD catheters. This may lead to the development of collaterals, and this leads to an increase in the flow and pressure in the esophageal plexus leading to the formation of varices, in the bronchial veins leading to hemoptysis, and in the intercostals veins leading to the formation of pleural effusion. The AV fistula aggravates these findings by further increasing both the flow and the pressure in the collateral circulation. 


\section{Grant Support}

None.

\section{Conflict of Interest}

Authors declare no conflict of interest.

\section{References}

1. Madan AK, Allmon JC, Harding M, Cheng SS, Slakey DP. Dialysis access-induced superior vena cava syndrome. Am Surg. 2002;68(10):904-906.

2. Akoglu H, Yilmaz R, Peynircioglu B, Arici M, Kirkpantur A, Cil B, Altun B, et al. A rare complication of hemodialysis catheters: superior vena cava syndrome. Hemodial Int. 2007;11(4):385-391.

3. Sriramnaveen P, Kumar VS, Kishore CK, Sai Naresh VV, Sivaramakrishna G, Manjusha Y, Lakshmi BV, et al. Delayed presentation of Superior Vena Cava Syndrome after hemodialysis catheter removal. Saudi J Kidney Dis Transpl. 2011;22(3):554-556.

4. Hamer R, Tagboto S. Superior vena caval obstruction following central venous cannulation. Nephrol Dial Transplant. 2004;19(1):258.

5. Wright RS, Quinones-Baldrich WJ, Anders AJ, Danovitch GM. Pleural effusion associated with ipsilateral breast and arm edema as a complication of subclavian vein catheterization and arteriovenous fistula formation for hemodialysis. Chest. 1994;106(3):950-952.

6. Chandra A, Tso R, Cynamon J, Miller G. Massive upper GI bleeding in a long-term hemodialysis patient. Chest. 2005;128(3):1868-1869, 1870-1873.

7. Hussein FA, Mawla N, Befeler AS, Martin KJ, Lentine KL. Formation of downhill esophageal varices as a rare but serious complication of hemodialysis access: a case report and comprehensive literature review. Clin Exp Nephrol. 2008;12(5):407-415.

8. Greenwell MW, Basye SL, Dhawan SS, Parks FD, Acchiardo SR. Dialysis catheter-induced superior vena cava syndrome and downhill esophageal varices. Clin Nephrol. 2007;67(5):325-330.

9. Calderwood AH, Mishkin DS. Downhill esophageal varices caused by catheter-related thrombosis. Clin Gastroenterol Hepatol. 2008;6(1):e1.

10. Gopaluni S, Warwicker P. Superior vena cava obstruction presenting with epistaxis, haemoptysis and gastro-intestinal haemorrhage in two men receiving haemodialysis with central venous catheters: two case reports. J Med Case Rep. 2009;3:6180.

11. Seelig MH, Oldenburg WA, Klingler PJ, Odell JA. Superior vena cava syndrome caused by chronic hemodialysis catheters: autologous reconstruction with a pericardial tube graft. J Vasc Surg. 1998;28(3):556-560.

12. Sheikh MA, Fernandez BB, Jr., Gray BH, Graham LM, Carman TL. Endovascular stenting of nonmalignant superior vena cava syndrome. Catheter Cardiovasc Interv. 2005;65(3):405-411. 